International Journal of Pure and Applied Mathematics

Volume 85 No. 2 2013, 395-404

ISSN: 1311-8080 (printed version); ISSN: 1314-3395 (on-line version)

url: http://www.ijpam.eu

doi: http://dx.doi.org/10.12732/ijpam.v85i2.15

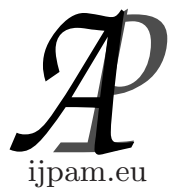

\title{
AN APPROXIMATE OPTIMAL SOLUTION FOR THE FUZZY VARIABLE LINEAR PROGRAMMING PROBLEM USING INTERIOR POINT TECHNIQUE
}

\author{
A. Nagoor Gani ${ }^{1}$, S.N. Mohamed Assarudeen ${ }^{2}$ \\ ${ }^{1,2} \mathrm{PG}$ and Research Department of Mathematics \\ Jamal Mohamed College (Autonomous) \\ Tiruchirappalli 20, INDIA
}

\begin{abstract}
In this paper an interior point technique is used to solve fuzzy variable linear programming problem to find the approximate optimal solution. In this method an initial starting interior fuzzy feasible point must be able to find from which we proceed to an improved interior fuzzy feasible point and we stop the iterations by some criteria.
\end{abstract}

AMS Subject Classification: 90C70, 90C05, 03E72

Key Words: fuzzy variable linear programming problem, triangular fuzzy number, interior point method

\section{Introduction}

The interior point method was invented by John von Neumann. Von Neumann suggested a new method of linear programming, using the homogeneous linear system of Gordan (1873) which was later popularized by Karmarkar's algorithm, developed by Narendra Karmarkar in 1984 for linear programming. A paper by karmarkar in 1984 [5] and substantial progress made since that time have

Received: January 28, 2013

(C) 2013 Academic Publications, Ltd.

$\S$ Correspondence author url: www.acadpubl.eu 
led to the field of modern interior point method for Linear problems. Unlike the family of simplex methods which approach the solution through a sequence of iterates that move from vertex to vertex along the edges on the boundary of the feasible space, the iterates generated by interior point algorithm approach the solution from the interior of the space. In this paper, an important primal affine scaling / dikins method is discussed in fuzzy environment.

In fuzzy decision making problems, the concept of maximizing decision was proposed by Bellman and Zadeh [2]. This concept was adopted to problems of mathematical programming by Tanaka et al. [10]. Zimmermann [11] presented a fuzzy approach to multiobjective linear programming problems. He also studied the duality relations in fuzzy linear programming. Fuzzy linear programming problem with fuzzy coefficients was formulated by Negoita [6] and called robust programming. Dubois and Prade [4] investigated linear fuzzy constraints. Tanaka and Asai [9] also proposed a formulation of fuzzy linear programming with fuzzy constraints and gave a method for its solution which bases on inequality relations between fuzzy numbers. Shaocheng [8] considered the fuzzy linear programming problem with fuzzy constraints and defuzzificated it by first determining an upper bound for the objective function. Further he solved the so-obtained crisp problem by the fuzzy decisive set method introduced by Sakawa and Yana [7].

This Paper has been organized as follows section 2 deals with some basic definitions of the fuzzy concept and fuzzy variable linear programming problem, section 3 explains the primal affine/Dikin's method with algorithm to solve fuzzy variable linear programming problem, in section 4 a numerical examples has been solved.

\section{Preliminaries}

Definition 1. Fuzzy Set: A fuzzy set $\tilde{A}$ is defined by $\tilde{A}=\left\{\left(\mathrm{x}, \mu_{A}(x)\right)\right.$ : $\left.\mathrm{x} \in \mathrm{A}, \mu_{A}(x) \in[0,1]\right\}$.In the pair $\left(\mathrm{x}, \mu_{A}(\mathrm{x})\right)$, the first element $\mathrm{x}$ belong to the classical set $\mathrm{A}$, the second element $\mu_{A}(x)$, belong to the interval $[0,1]$, called Membership function

Definition 2. Triangular Fuzzy Number: It is a fuzzy number represented with three points as follows: $\tilde{A}=\left(a_{1}, a_{2}, a_{3}\right)$. This representation in interpreted as membership functions 


$$
\mu_{A}(x)=\left\{\begin{array}{lll}
0 & \text { for } & x<a_{1} \\
\frac{x-a_{1}}{a_{2}-a_{1}} & \text { for } & a_{1} \leq x \leq a_{2} \\
\frac{a_{3}-x}{a_{3}-a_{2}} & \text { for } \quad a_{2} \leq x \leq a_{3} \\
0 & \text { for } & x>a_{3}
\end{array}\right.
$$

\subsection{Operation of Triangular Fuzzy Number using Function Principle}

The following are the four operations that can be performed on triangular fuzzy numbers: Let $\tilde{A}=\left(a_{1}, a_{2}, a_{3}\right)$ and $\tilde{B}=\left(b_{1}, b_{2}, b_{3}\right)$

1. Addition: $\tilde{A}+\tilde{B}=\left(a_{1}+b_{1}, a_{2}+b_{2}, a_{3}+b_{3}\right)$.

2. Subtraction: $\tilde{A}-\tilde{B}=\left(a_{1}-b_{3}, a_{2}-b_{2}, a_{3}-b_{1}\right)$.

3. Multiplication: $\tilde{A} \bullet \tilde{B}=\left(\min \left(a_{1} b_{1}, a_{1} b_{3}, a_{3} b_{1}, a_{3} b_{3}\right)\right.$, $\left.a_{2} b_{2}, \max \left(a_{1} b_{1}, a_{1} b_{3}, a_{3} b_{1}, a_{3} b_{3}\right)\right)$.

4. Scalar Multiplication: $\mathrm{k}(\tilde{A})=\left(k a_{1}, k a_{2}, k a_{3}\right)$ if $\mathrm{k}$ is positive and $\left(k a_{3}\right.$, $\left.k a_{2}, k a_{1}\right)$ if $\mathrm{k}$ is negative.

5. Division: $\tilde{A} / \tilde{B}=\left(\min \left(a_{1} / b_{1}, a_{1} / b_{3}, a_{3} / b_{1}, a_{3} / b_{3}\right), a_{2} / b_{2}\right.$, $\left.\max \left(a_{1} / b_{1}, a_{1} / b_{3}, a_{3} / b_{1}, a_{3} / b_{3}\right)\right)$.

\subsection{Fuzzy variable Linear Programming Model}

Consider the following fuzzy variable linear programming model:

$\tilde{Z}=c^{T} \tilde{x}$

Constraints of the form

$a_{j}^{T} \tilde{x}=\tilde{b}_{j}, \mathrm{j}=1,2, \ldots, \mathrm{m}$

and the nonnegative conditions of the fuzzy variables $\tilde{x} \geq \tilde{0}$ are also included in the form $-\tilde{x} \leq \tilde{0}$. where $c^{T}=\left(c_{1}, \ldots, c_{n}\right)$ is an n-dimensional constant vector, $\tilde{x}=\left(\tilde{x}_{i}\right), \mathrm{i}=1,2, . . \mathrm{n}$ and $\tilde{b}_{j}$ are non-negative fuzzy variable vectors such that $\tilde{x}_{i}$ and $\tilde{b}_{j} \in F(R)$ for all $1 \leq i \leq n, 1 \leq j \leq m$, is called a fuzzy variable linear programming (FVLP) problem.

\section{Primal Affine Scaling Method}

The primal affine scaling method was proposed in [1] as modification of Karmarker's interior point algorithm[5] for LP problems. Through conceptually 
simple, the method has been found effective partically for large scale problems.

Consider the standard fuzzy variable linear programming problems and let $\tilde{x}^{0}$ be a strictly interior fuzzy feasible point. There are two major steps of the primal affine scaling method involve moving $\tilde{x}^{0}$ along a projected steepest descent direction for minimization problem(steepest ascent direction for maximization) and scaling the resulting point to center it in the feasible region in the transformed space.

\subsection{Maintaining the Feasibility}

A feasible solution $\tilde{x}^{0}$ must satisfy $A \tilde{x}^{0}=\tilde{b}$. Hence the only feasible interior point $\tilde{x}=\tilde{x}^{t}$ are those satisfy $A \tilde{x}^{t}=\tilde{b}$ and $\tilde{x}^{t} \geq \tilde{0}$. As a result, when ever we move from a point $\tilde{x}^{t}$ to $\tilde{x}^{t+1}=\tilde{x}^{t}+\alpha p^{t}$ with $\alpha>0$ and $p^{t}=P c$ we need to satisfy $A \tilde{x}^{t+1}=\tilde{b}$ and $\tilde{x}^{t+1}>0$. This implies that

$A \tilde{x}^{t+1}=A\left(\tilde{x}^{t+1}\right)=A\left(\tilde{x}^{t}+\alpha p^{t}\right)=A \tilde{x}^{t}+\alpha A p^{t}=\tilde{b}+\alpha A p^{t}$

Since $\alpha \neq 0$, the above equation implies that in order to stay feasible $p^{t}$ must satisfy $\alpha p^{t}=0$.

In otherwords $p^{t} \neq 0$ must be orthogonal to the rows of $\mathrm{A}$. A vector direction $p^{t}$ that satisfied $A p^{t}=0$ is said to belong to the null space of $\mathrm{A}$. The projection Matrix $P=I-A^{T}\left(A A^{T}\right)^{-1} A$ will transform any vector $\mathrm{v}$ into $\mathrm{Pv}=\mathrm{p}$ and $\mathrm{p}$ will be in the null space of $\mathrm{A}$ because $\mathrm{AP}=0$ is a zero matrix. Hence in order to maintain feasibility every move from $\tilde{x}^{t}$ to $\tilde{x}^{t+1}$ must be in the null space of A. ie $p^{t}=P v$ for some vector $\mathrm{v}$.

The move direction of most rapid improvement for linear objective Maximize $c \tilde{x}=\sum_{j} c_{j} \tilde{x}_{j}$ is the objective function vector $p^{t}=P c$. For Min $c \tilde{x}$ it is $p^{t}=-P c$. However the condition that we must maintain feasibility implies that we move in the direction of steepest descent(ascent) projected into the null space of $\mathrm{A}$.

That is, for Minimization (Maximization) $p^{t}=-P c(P c)$

Such direction is called projected gradient direction. To see such a direction is a direction of decrease (increase)let for minimization $\tilde{x}^{t+1}=\tilde{x}^{t}+\alpha p^{t}$ with $\alpha>0$ and $p^{t}=-P c$

$c^{T} \tilde{x}^{t+1}=c^{T}\left(\tilde{x}^{t}+\alpha p^{t}\right)=c^{T} \tilde{x}^{t}-\alpha c^{T} P c \leq c^{T} \tilde{x}^{t}$

For maximimation $\tilde{x}^{t+1}=\tilde{x}^{t}+\alpha p^{t}$ with $\alpha>0$ and $p^{t}=P c$

$c^{T} \tilde{x}^{t+1}=c^{T}\left(\tilde{x}^{t}+\alpha p^{t}\right)=c^{T} \tilde{x}^{t}+\alpha c^{T} P c \geq c^{T} \tilde{x}^{t}$

Where the last inequality follows because $\mathrm{P}$ has a property that $P=P^{T} P$ and therefore $c^{T} P c=c^{T} P^{T} P c=(P c)^{T}(P c) \geq 0$. 


\subsection{Staying in the Interior}

Next staying in the interior ie $\tilde{x} \geq \tilde{0}$ by limiting the amount of movement $\alpha>0$ in the direction $p^{t}$. The quantity $\alpha$ called the steplength, is chosen to ensure feasibility of the non negativity constraints. Here we want to move some portion of the way towards the boundary. Based on the experience of others on practical problem moving $50 \%$ to $90 \%$ of the way is a simple good rule for Primal Affine Scaling/ Dikin's Method.

\subsection{Centering}

Here transforming the space so that an interior point $\tilde{x}^{t}$ is the center point in the transformed space. The basic idea is change the scaling of the solution so that each component is equidistant from the constraints boundaries in the transformed feasible region; for example after rescaling $\tilde{x}^{t}=\tilde{e}$, where $\tilde{e}=$ $(\tilde{1}, \tilde{1}, \ldots, \tilde{1})^{T}$. Let $\tilde{S}=\operatorname{Diag}\left(\tilde{x}^{t}\right)$ be the diagonal matrix having the components of the currents iterate $\tilde{x}^{t}$ as the diagonal elements. The simplest such scheme is rescale $\tilde{x}$ by setting $\tilde{x}=\tilde{S} \tilde{\bar{x}}$ so that $\tilde{x}^{t}=\tilde{e}$. Sub $\tilde{x}=\tilde{S} \tilde{\bar{x}}$ in FVLP $\operatorname{Max}(\operatorname{Min}) \tilde{\bar{C}}^{T} \tilde{x}=\tilde{Z}$

s.t $\tilde{D} \tilde{x}=\tilde{b} ; \quad \tilde{D}=m * n$

$\tilde{x}>\tilde{0}$

Where $\tilde{D}=A \tilde{S}$ and $\tilde{\bar{C}}=\tilde{S} c$

As a result of this transformation clearly projection matrix $\tilde{\bar{P}}=\tilde{I}-\tilde{D}^{T}\left(\tilde{D} \tilde{D}^{T}\right)^{-1} \tilde{D}$

Hence at each iteration t, we rescale $\tilde{x}^{t}$ to $\tilde{x}=\tilde{e}$ and move to $\tilde{x}^{t+1}$ in the negative (positive for Max) projected gradient direction $-\tilde{\bar{P}} \tilde{\bar{C}}(\tilde{\bar{P}} \tilde{\bar{C}}$ for max) thus

$\tilde{x}^{t+1}=\tilde{e}-\alpha \tilde{\bar{P}} \tilde{\bar{C}}$ for min

$\tilde{x}^{t+1}=\tilde{e}+\alpha \tilde{\bar{P}} \tilde{\bar{C}}$ for $\max$

Finally we rescale back to the original space by setting $\tilde{x}^{t+1}=\tilde{S} \tilde{x}^{t+1}$. Next we repeat the iterative process replacing $\tilde{S}$ by $\tilde{S}=\operatorname{Diag}\left(\tilde{x}^{t+1}\right)$.

\subsection{Stopping Rule}

The simple rule typically followed is to stop with an approximate optimal solution when the difference between iterates $\tilde{x}^{t+1}$ and $\tilde{x}^{t}$ is sufficiently small in the original space. 


\subsection{Algorithm}

Assume an initial feasible interior point solution $\tilde{x}=\tilde{x}>0 \&$ satisfying $\mathrm{A} \tilde{x}=\tilde{b}$

1. Initially set $\mathrm{t}=0$

2. Create $\tilde{S}:$ Set $\tilde{S}=\operatorname{Diag}\left(\tilde{x}^{t}\right)$

3. Centering Transformation compute $\tilde{D}=A \tilde{S}$ and $\tilde{\bar{C}}=\tilde{S} c$

4. Now determine the projection matrix $\tilde{\bar{P}}=\tilde{I}-\tilde{D}^{T}\left(\tilde{D} \tilde{D}^{T}\right)^{-1} \tilde{D}$

5. Compute steepest descent (ascent) direction set $\tilde{p}^{t}=-\tilde{\bar{P}} \tilde{\bar{C}}$ for min $\tilde{p}^{t}=$ $\tilde{\bar{P}} \tilde{\bar{C}}$ for $\max$

6. Now find $\tilde{\theta}=-\min \left(\tilde{p}^{t}\right)$ (use center b value of TFN $(\mathrm{a}, \mathrm{b}, \mathrm{c})$ )

7. Test for unbound objective if $\tilde{\theta}<\tilde{0}$ report the objective as being unbound \& stop.

8. Obtain $\tilde{x}^{t+1}=\tilde{e}+\frac{\alpha}{\hat{\theta}} \tilde{p}^{t}$ where $\tilde{e}=(\tilde{1}, \tilde{1}, . ., \tilde{1})^{T}$ and $\alpha$ is set to a number between 0 \& 1

9. Obtain new solution $\tilde{x}^{t+1}=\tilde{S} \tilde{x}^{t+1}$

10. Termination rule: if $\tilde{x}^{t+1}$ is close to $\tilde{x}^{t}$ report $\tilde{x}^{t+1}$ as close to optimal and stop.

11. If the difference is big, set $\mathrm{t}=\mathrm{t}+1$ and goto step 2 .

\section{Numerical Example}

$$
\text { Maximize } \quad \tilde{Z}=5 \tilde{x}_{1}+3 \tilde{x}_{2}
$$

Subject to constraints

$$
\begin{gathered}
3 \tilde{x}_{1}+5 \tilde{x}_{2} \leq(14,15,16) \\
5 \tilde{x}_{1}+2 \tilde{x}_{2} \leq(9,10,11) \\
\tilde{x}_{1}, \tilde{x}_{2} \geq \tilde{0}
\end{gathered}
$$


Transform inequality into equality form

$3 \tilde{x}_{1}+5 \tilde{x}_{2}+\tilde{x}_{3}=(14,15,16)$

$5 \tilde{x}_{1}+2 \tilde{x}_{2}+\tilde{x}_{4}=(9,10,11)$

Solution

Taking a feasible solution approximately which is an interior point of the solution space.

$\tilde{x}^{0}=((1,1,1),(2,2,2),(1,2,3),(0,1,2)) \& \tilde{Z}^{0}=(11,11,11)$

Now form $\tilde{S}$ matrix $=\left(\operatorname{Diag}\left(\tilde{x}^{0}\right)\right)$

$$
\tilde{S}=\left(\begin{array}{llll}
(1,1,1) & & & \\
& (2,2,2) & & \\
& & (1,2,3) & \\
& & & (0,1,2)
\end{array}\right)
$$

Calculate

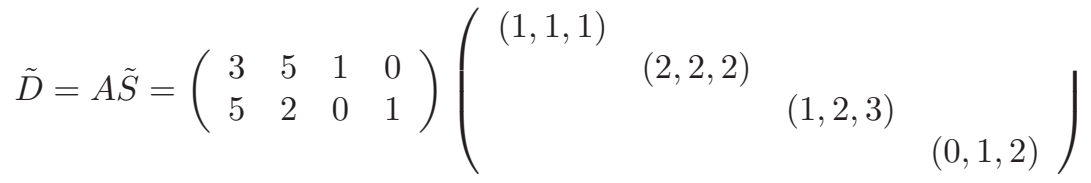

$$
\begin{aligned}
& \tilde{D}=\left(\begin{array}{llll}
(3,3,3) & (10,10,10) & (1,2,3) & (0,0,0) \\
(5,5,5) & (4,4,4) & (0,0,0) & (0,1,2)
\end{array}\right)
\end{aligned}
$$

Calculate

$$
\begin{gathered}
\tilde{\bar{C}}=\tilde{S} c\left(\begin{array}{ccc}
(1,1,1) & & \\
& (2,2,2) & \\
& (1,2,3) & \\
& & (0,1,2)
\end{array}\right)\left(\begin{array}{l}
5 \\
3 \\
0 \\
0
\end{array}\right) \\
\tilde{\bar{C}}=\left(\begin{array}{l}
(5,5,5) \\
(6,6,6) \\
(0,0,0) \\
(0,0,0)
\end{array}\right)
\end{gathered}
$$

Calculate Projection Matrix $\tilde{\bar{P}}=\tilde{I}-\tilde{D}^{T}\left(\tilde{D} \tilde{D}^{T}\right)^{-1} \tilde{D}$

$$
\begin{gathered}
\tilde{D} \tilde{D}^{T}=\left(\begin{array}{llll}
(3,3,3) & (10,10,10) & (1,2,3) & (0,0,0) \\
(5,5,5) & (4,4,4) & ((0,0,0) & (0,1,2)
\end{array}\right)\left(\begin{array}{ll}
(3,3,3) & (5,5,5) \\
(10,10,10) & (4,4,4) \\
(1,2,3) & (0,0,0) \\
(0,0,0) & (0,1,2)
\end{array}\right) \\
=\left(\begin{array}{ll}
(110,113,118) & (55,55,55) \\
(55,55,55) & (41,42,45)
\end{array}\right) \\
=\frac{1}{(1485,1721,2285)}\left(\begin{array}{ll}
(41,42,45) & -(55,55,55) \\
-(55,55,55) & (110,113,118)
\end{array}\right)
\end{gathered}
$$




$$
\begin{aligned}
& =\left(\begin{array}{cc}
(0.018,0.024,0.030) & -(0.024,0.032,0.037) \\
-(0.024,0.032,0.037) & (0.048,0.066,0.079)
\end{array}\right) \\
& \tilde{D}^{T}\left(\tilde{D} \tilde{D}^{T}\right)^{-1}=\left(\begin{array}{ll}
(3,3,3) & (5,5,5) \\
(10,10,10) & (4,4,4) \\
(1,2,3) & (0,0,0) \\
(0,0,0) & (0,1,2)
\end{array}\right)\left(\begin{array}{ll}
(0.018,0.024,0.030) & -(0.024,0.032,0.037) \\
-(0.024,0.032,0.037) & (0.048,0.066,0.079)
\end{array}\right) \\
& =\left(\begin{array}{ll}
-(0.03,0.088,0.131) & (0.129,0.234,0.323) \\
(0.032,0.112,0.204) & (-0.076,-0.056,0.178) \\
(0.018,0.048,0.09) & -(0.024,0.064,0.111) \\
-(0,0.032,0.074) & (0,0.066,0.158)
\end{array}\right) \\
& \tilde{D}^{T}\left(\tilde{D} \tilde{D}^{T}\right)^{-1} \tilde{D}=\left(\begin{array}{ll}
-(0.03,0.088,0.131) & (0.129,0.234,0.323) \\
(0.032,0.112,0.204) & (-0.076,-0.056,0.178) \\
(0.018,0.048,0.09) & -(0.024,0.064,0.111) \\
-(0,0.032,0.074) & (0,0.066,0.158)
\end{array}\right)\left(\begin{array}{llll}
(3,3,3) & (10,10,10) & (1,2,3) & (0,0,0) \\
(5,5,5) & (4,4,4) & (0,0,0) & (0,1,2)
\end{array}\right) \\
& =\left(\begin{array}{llll}
(0.252,0.906,1.525) & (-0.794,0.056,0.992) & -(0.03,1.76,0.393) & (0,0.234,0.646) \\
(-0.284,0.056,1.502) & (0.016,0.896,2.752) & (0.032,0.224,0.612) & (-0.152,-0.056,0.356) \\
-(-0.15,0.176,0.501) & (-0.264,0.224,0.804) & (0.018,0.096,0.27) & -(0,0.064,0.222) \\
-(0.222,0.234,0.79) & (-0.74,-0.056,0.296) & -(0,0.64,0.222) & (0,0.066,0.316)
\end{array}\right) \\
& \tilde{\bar{P}}=\tilde{I}-\tilde{D}^{T}\left(\tilde{D} \tilde{D}^{T}\right)^{-1} \tilde{D} \\
& =\left(\begin{array}{llll}
(-0.525,0.094,0.748) & -(-0.794,0.056,0.992) & (0.03,1.76,0.393) & -(0,0.234,0.646) \\
-(-0.284,0.056,1.502) & (-1.752,0.104,0.84) & -(0.032,0.224,0.612) & -(-0.152,-0.056,0.356) \\
(-0.15,0.176,0.501) & -(-0.264,0.224,0.804) & (0.73,0.904,0.982) & (0,0.064,0.222) \\
-(0.222,0.234,0.79) & -(-0.74,-0.056,0.296) & (0,0.64,0.222) & (0.684,0.934,1)
\end{array}\right)
\end{aligned}
$$

Now the problem is maximize

Therefore $\tilde{p}^{t}=\tilde{\bar{P}} \tilde{\bar{C}}$

where

$$
\tilde{\bar{C}}=\left(\begin{array}{c}
(5,5,5) \\
(6,6,6) \\
((0,0,0) \\
(0,0,0)
\end{array}\right)
$$

$$
\begin{gathered}
\tilde{\bar{P}}=\left(\begin{array}{llll}
(-0.525,0.094,0.748) & -(-0.794,0.056,0.992) & (0.03,1.76,0.393) & -(0,0.234,0.646) \\
-(-0.284,0.056,1.502) & (-1.752,0.104,0.84) & -(0.032,0.224,0.612) & -(-0.152,-0.056,0.356) \\
(-0.15,0.176,0.501) & -(-0.264,0.224,0.804) & (0.73,0.904,0.982) & (0,0.064,0.222) \\
-(0.222,0.234,0.79) & -(-0.74,-0.056,0.296) & (0,0.64,0.222) & (0.684,0.934,1)
\end{array}\right) \\
\tilde{p}^{t}=\left(\begin{array}{ll}
(-8.577,0.134,8.504) \\
(-18.022,0.344,6.46) \\
(-5.574,-0.464,4.089) \\
(-5.726,-0.834,3.33)
\end{array}\right)
\end{gathered}
$$

$\tilde{\theta}=-\min \left(\tilde{p}^{t}\right)=(-3.33,0.834,5.726)$

$$
\tilde{\bar{x}}^{1}=\left(\begin{array}{c}
(1,1,1) \\
(1,1,1) \\
(1,1,1) \\
(1,1,1)
\end{array}\right)+\frac{\alpha}{\tilde{\theta}}\left(\begin{array}{c}
(-8.577,0.134,8.504) \\
(-18.022,0.344,6.46) \\
(-5.574,-0.464,4.089) \\
(-5.726,-0.834,3.33)
\end{array}\right)
$$




$$
\begin{gathered}
=\left(\begin{array}{c}
(1,1,1) \\
(1,1,1) \\
(1,1,1) \\
(1,1,1)
\end{array}\right)+\left(\begin{array}{c}
(-1.28,0.08,1.28) \\
(-1.57,0.21,2.71) \\
(-0.61,-0.27,0.84) \\
(-0.5,-0.5,0.86)
\end{array}\right)=\left(\begin{array}{c}
(-0.28,1.08,2.28) \\
(-0.57,1.21,3.71) \\
(0.39,0.73,1.84) \\
(0.5,0.5,1.86)
\end{array}\right) \\
\tilde{x}^{1}=\tilde{S} \tilde{\bar{x}}^{1}=\left(\begin{array}{ll}
(1,1,1) \\
(2,2,2)
\end{array}\right)\left(\begin{array}{c}
(-0.28,1.08,2.28) \\
(-0.57,1.21,3.71) \\
(0.39,0.73,1.84) \\
(0.5,0.5,1.86)
\end{array}\right) \\
=\left(\begin{array}{c}
(-0.28,1.08,2.28) \\
(-1.14,2.42,7.42) \\
(0.39,1.46,5.52) \\
(0,0.5,3.72)
\end{array}\right)
\end{gathered}
$$

There is not big difference between $\tilde{x}^{1}$ and $\tilde{x}^{0}$ it is the approximate optimal solution for fuzzy variable linear programming now $\tilde{Z}^{1}=(-4.82,12.66,33.66)$.

\section{Conclusion}

In this paper, the new method solving fuzzy variable linear programming problem is discussed. Here we use an interior point technique to solve the FVLPP. In simplex method we always obtain the solution on the boundary line, from that solution some fuzzy variables of the LPP may be zero. But here we obtain the solution for the fuzzy variable from the interior point technique is always not equal zero.

\section{References}

[1] Barnes, E. R. "A variation on Karmarkar's algorithm for solving linear programming problems", Math. Programming, vol. 36, pp. 174-182, 1986. doi:10.1007/BF02592024.

[2] Bellman, R.E., Zadeh, L.A., "Decision-making in a fuzzy environment", Management Science 17 (1970) B141-B164. doi:10.1287/mnsc.17.4.B141.

[3] Dantzig, George B., Thapa, Mukund N. (2003). Linear Programming 2: Theory and Extensions. Springer-Verlag.ISBN 978-0-387-98613-5

[4] Dubois, D., Prade, H., "System of linear fuzzy constraints", Fuzzy Sets and Systems, Volume 3, Issue 1, January 1980, Pages 37-48. doi.org/10.1016/01650114(80)90004-4.

[5] Karmarkar, N.K. " A new polynomial time algorithm for linear programming problem", Combinatorica, vol. 4, pp.373-395,1984. doi:10.1007/BF02579150 
[6] Negoita, C.V.: "Fuzziness in management", OPSA/TIMS, Miami (1970).

[7] Sakawa, M., Yano, H.: "Interactive decision making for multi-objective linear fractional programming problems with fuzy parameters", Cybernetics Systems 16 (1985) 377-394. doi:10.1080/01969728508927781

[8] Shaocheng, T.: "Interval number and Fuzzy number linear programming", Fuzzy Sets and Systems 66 (1994) 301-306. doi:10.1016/0165-0114(94)90097-3.

[9] Tanaka, H. and Asai, K.," Fuzzy linear programming problems with fuzzy numbers", Fuzzy Sets and Systems 13 (1984) 1-10. doi.org/10.1016/01650114(84)90022-8

[10] Tanaka, H., Okuda, T.and Asai, K.,"On fuzzy mathematical programming", J. Cybernetics 3(1984) 37-46. doi:10.1080/01969727308545912.

[11] Zimmermann, H.J.,"Fuzzy mathematical programming", Comput. \& Ops. Res. Vol. 10 No 4 (1983) 291-298. 